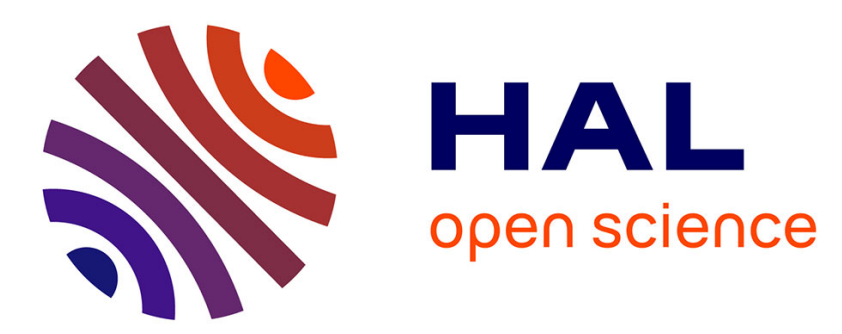

\title{
Constrained Model Predictive Control of a Skid-Steering Mobile Robot
}

Federico Bribiesca Argomedo, Nicolas Marchand, Olivier Sename

\section{To cite this version:}

Federico Bribiesca Argomedo, Nicolas Marchand, Olivier Sename. Constrained Model Predictive Control of a Skid-Steering Mobile Robot. ECC 2009 - European Control Conference, Aug 2009, Budapest, Hungary. hal-00368511

\section{HAL Id: hal-00368511 https://hal.science/hal-00368511}

Submitted on 20 Feb 2015

HAL is a multi-disciplinary open access archive for the deposit and dissemination of scientific research documents, whether they are published or not. The documents may come from teaching and research institutions in France or abroad, or from public or private research centers.
L'archive ouverte pluridisciplinaire $\mathbf{H A L}$, est destinée au dépôt et à la diffusion de documents scientifiques de niveau recherche, publiés ou non, émanant des établissements d'enseignement et de recherche français ou étrangers, des laboratoires publics ou privés. 


\title{
Constrained Model Predictive Control of a Skid-Steering Mobile Robot
}

\author{
Federico Bribiesca Argomedo, Nicolas Marchand and Olivier Sename \\ GIPSA-lab, Department of Control Systems \\ Grenoble INP / ENSE3 - BP 46. 38402 Saint Martin d'Hères Cedex. Grenoble, France. \\ \{Nicolas.Marchand, Olivier. Sename\}@grenoble-inp. fr
}

\begin{abstract}
In this paper, a kinematic model of a four-wheel skid-steering mobile robot is presented and a receding horizon stabilizing control law for the system is developed, based on the optimization of a quadratic cost function on the system states and control inputs. Global asymptotic stability of the nominal system with actuator saturation constraints is analytically proven and a simple dynamical model is constructed for validation purposes. The robustness and performance of the controller were tested under simulation on both models and the results are presented and discussed.

Index Terms - Skid-steering, Non-holonomic systems, Global stabilization, Receding Horizon Control, Quadratic optimization, Actuator saturations.
\end{abstract}

\section{INTRODUCTION}

The control of non-holonomic systems has become a widespread research topic since many underactuated mechanical systems, such as vehicles and robotic manipulators, fall within this category. Extensive literature can be found on the subject (see e.g. $[9,11,12,18,19]$ ).

The trajectory tracking problem, i.e. having the system follow a desired path, has been tackled in several different ways. The first main approach consists in high-level controllers implemented using tools such as fuzzy logic $[13,20]$ but they offer, in general, little analytical performance guarantees. The second important approach consists in using a Control Lyapunov Function to construct an appropriate (i.e. stable) controller. While quite popular, e.g. $[3,7,13]$, it has the inconvenient of being unable to guarantee stability if the reference does not preserve some level of activity. Commonly, it requires the velocity of the reference to be different from zero at all times.

To achieve stabilization with a static reference, three main techniques are often used. The first one consists in a timevarying feedback in the form of a kinematic oscillator like those used in $[10,15]$. The second technique is the use of discontinuous, time-invariant stabilization using piecewise continuous controllers; see [2,14]. Finally, the third approach is Model Predictive Control (MPC, or Receding Horizon Control) which uses piecewise constant controls. See for instance $[1,5,6,16,17]$.

We will focus on the MPC approach which consists in solving, for each sample period, a finite-horizon, optimal control problem for the open-loop system. This generates a viable trajectory for the nominal system on which only the first control value will be applied. By using a varying-length horizon instead of a fixed one, final state constraints can

This work was supported by the Teromac project be included. This ensures that, at the end of the prediction horizon, the system will have reached a set of allowed states. Part of the work developed was directed at proving that the proposed MPC scheme is always able to find a solution to the optimal control problem under the given constraints and for finite errors in position with non-zero actuator saturation levels.

The use of MPC was favored due to the possible inclusion of hard-constraints (such as actuator saturations and possibly forbidden states) as well as energetic considerations into the calculation of the control profiles. Since a complete control profile is calculated at each time interval, the controller is also inherently robust with respect to previous disturbances. The main problem tackled was assuring the robust feasibility of the proposed algorithm (this is, that it will be able to find a path at all times even when a disturbance seriously disrupts the state of the system). The most evident limitation of this approach is the associated computational cost; but efficient routines exist to solve quadratic optimization problems and the system under consideration is not required to be extremely fast.

The main objectives of this paper are to present a kinematic MPC controller along with an analytical proof of its stability and to test its robustness w.r.t. unmodeled dynamics. It will also be shown that the particular velocity profile chosen for the angle subsystem permits bounding the prediction horizon required to solve the optimization problem. This is an improvement over previous approaches since it simplifies the stability proof and implies the feasibility of finite-time stabilization under the given conditions.

In the first section, we will develop a kinematic model for the skid-steering vehicle. In the second section, the control problem will be stated and the corresponding open-loop and closed-loop control laws will be introduced. Also a sketch of the proof of asymptotic stability for the nominal system will be presented. In the third section, a dynamic model used for controller validation under simulation will be obtained. Finally, simulation results and conclusions will be presented.

\section{Kinematic ModeL}

We now consider the well-known unicycle model $[3,7$, 10,13 ] for the kinematic analysis, based on the hypothesis of negligible longitudinal slip in all four wheels and a rigid structure. 
The equation describing the evolution of the system is:

$$
\dot{\mathbf{q}}=\left[\begin{array}{c}
\dot{x} \\
\dot{y} \\
\dot{\theta}
\end{array}\right]=\left[\begin{array}{cc}
\cos \theta & 0 \\
\sin \theta & 0 \\
0 & 1
\end{array}\right]\left[\begin{array}{l}
u \\
v
\end{array}\right]
$$

Where $u$ is the linear velocity of the system and $v$ its angular velocity.

Since it is known that this system does not satisfy the Brockett conditions, there exists no stabilizing continuous state feedback [4]. More details on the obstruction can be found in [19] and [18].

\section{A. Stabilizing Control Law Synthesis}

Given a position reference $\mathbf{q}_{\text {ref }}$, considered constant in the case of stabilization, it is possible to define the position error of the robot in the relative frame of reference $x_{r e l} G y_{r e l}$ as follows:

$$
\mathbf{q}_{e}=\left[\begin{array}{l}
x_{e_{r e l}} \\
y_{e_{r e l}} \\
\theta_{e_{r e l}}
\end{array}\right]=\left[\begin{array}{ccc}
\cos \theta & \sin \theta & 0 \\
-\sin \theta & \cos \theta & 0 \\
0 & 0 & 1
\end{array}\right]\left[\begin{array}{l}
x_{r e f}-x \\
y_{r e f}-y \\
\theta_{r e f}-\theta
\end{array}\right]
$$

To simplify the notation, this vector (in the relative coordinate system) will be referred to as the error vector; thus avoiding the inclusion of the "rel" subindexes.

Based on the approach proposed in $[1,5,16]$ we will set to develop a model predictive receding horizon stabilizing control. Since finding the optimal solution of the global problem is quite complex; we will separate it into two subsystems: $(\theta)$ and $(x, y)^{T}$, and then find an appropriate trajectory for each one. We will not in general obtain a globally optimal path; but it can be computed faster. Furthermore, we can obtain interesting energetic properties while taking into account from the beginning the boundedness of control inputs.

1) Open-loop Control Problem: Considering the system as defined in (1) our goal is then to find, at time $t_{0}$, an openloop control strategy that drives it from an initial state $\mathbf{q}_{0}$ to a final state $\mathbf{q}_{f}$ in a time $\Delta t>0$. Therefore, we will define the control law in the interval $\left[t_{0}, t_{0}+\Delta t\right]$. We also consider a sampling period $T$ such that $\Delta t=N T ; N \in \mathbb{Z}^{+}, T \in \mathbb{R}^{+}$. By taking the control inputs as constant during each period $T$, we can fully define the control law with two vectors:

$$
\begin{aligned}
U & =\left[\begin{array}{llll}
u_{0} & u_{1} & \ldots & u_{N-1}
\end{array}\right]^{T} \\
V & =\left[\begin{array}{llll}
v_{0} & v_{1} & \ldots & v_{N-1}
\end{array}\right]^{T}
\end{aligned}
$$

Where $u_{i}, v_{j}$ verify that $-u_{\max } \leq u_{i} \leq u_{\max },-v_{\max } \leq$ $v_{j} \leq v_{\text {max }} ; \forall i, j$. We will now deviate from the procedure used in other papers by defining a simpler structure for the control vector $U$ that allows us to analytically ensure some properties for the found paths.

For the control of the $\theta$ subsystem, we want to find $U$ such that the angle is driven from an initial condition $\theta_{0}$ to a final one $\theta_{f}$. Without loss of generality we will consider $\theta_{f} \equiv 0$.

Since the controls are defined as constant during each period $T$, the system, with $\zeta=(x, y)^{T}$ accepts the following discretization:

$$
\begin{aligned}
\zeta_{k+1} & =\zeta_{k}+\left[\begin{array}{c}
\sin \left(\theta_{k}+v_{k} T\right)-\sin \theta_{k} \\
\cos \theta_{k}-\cos \left(\theta_{k}+v_{k} T\right)
\end{array}\right] u_{k} \\
\theta_{k+1} & =\theta_{k} \oplus v_{k} T
\end{aligned}
$$

Where $\oplus$ denotes a sum of angles, with the result being always in $(-\pi, \pi]$. It follows then that the value of $\theta$ at the final time can be expressed as:

$$
\theta_{N}=\theta_{0} \oplus\left[\begin{array}{llll}
T & T & \ldots & T
\end{array}\right] V
$$

Where we will require $\theta_{N}=\theta_{f}$. To avoid singularities where the angle vanishes too quickly (therefore loosing all $y$ controllability) we will force $\theta$ to drift initially for $\delta$ sampling periods. We can define then, for a given set of parameters $\left(N, T, \theta_{0}, \delta, \alpha\right)$, a single well-characterized trajectory for the $\theta$ subsystem whenever the corresponding $\left|\frac{\Delta \theta}{T(N-\delta)}\right| \leq v_{\max }$ as follows:

$$
\begin{aligned}
& \hat{V}\left(N, T, \theta_{0}, \delta, \alpha\right)=\left[\begin{array}{llll}
v_{0} & v_{1} & \ldots & v_{N-1}
\end{array}\right]^{T} \\
& v_{0}, \ldots, v_{\delta-1}=\alpha v_{\max } \\
& v_{\delta}, \ldots, v_{N-1}=\frac{-\Delta \theta}{T(N-\delta)} \\
& \Delta \theta=\left(\theta_{0} \oplus\left(\delta T \alpha v_{\max }\right)\right)
\end{aligned}
$$

Notice that no viable trajectories will be found if $\left|\frac{\Delta \theta}{T(N-\delta)}\right|>v_{\max }$.

Once the $V$ vector is determined, the evolution of the $\zeta$ subsystem can be found to be, from (4) and (3):

$$
\zeta_{N}=\left[\begin{array}{ll}
1 & 0 \\
0 & 1
\end{array}\right] \zeta_{0}+\left[\begin{array}{llll}
s_{0} & s_{1} & \ldots & s_{N-1} \\
c_{0} & c_{1} & \ldots & c_{N-1}
\end{array}\right] U
$$

Where the coefficients $s_{i}, c_{j}$ are defined as follows:

$$
\begin{aligned}
s_{i} & \equiv \frac{1}{v_{i}}\left[\sin \left(\theta_{i}+v_{i} T\right)-\sin \theta_{i}\right] \\
c_{j} & \equiv \frac{1}{v_{j}}\left[\cos \theta_{j}-\cos \left(\theta_{j}+v_{j} T\right)\right]
\end{aligned}
$$

If $v_{i, j}=0$ the coefficients are calculated using L'Hôpital's rule as:

$$
s_{i}=T \cos \theta_{i} \quad c_{j}=T \sin \theta_{i}
$$

The necessary computation of the $\theta$ trajectory from the control profile can be done by applying equation (4).

Now we can define a path for the $(x, y)$ subsystem as the solution of the following quadratic optimization problem:

$$
\begin{aligned}
U\left(N, T, \zeta_{0}, \hat{V}\right) & =\left\{\left[\begin{array}{llll}
u_{0} & u_{1} & \ldots & u_{N-1}
\end{array}\right]^{T} \mid \zeta_{N}=0\right\} \\
\hat{U} & =\arg \min _{U} \sum_{i=0}^{N-1}\left(\zeta_{i}^{T} O \zeta_{i}+p u_{i-1}^{2}\right)
\end{aligned}
$$

Where $O, p>0$ are weighting parameters; the control inputs are subject to the saturation constraints previously defined for all $u_{i}$; and the final state, calculated with the formula stated in (7), is equal to the desired $\zeta_{N} \equiv 0$. 
Now we can define a set of profiles constituted as:

$$
\begin{aligned}
& \mathcal{C}\left(N_{\max }, T, \theta_{0}, \zeta_{0}, \delta_{\max }, \beta\right)= \\
&\left\{(\hat{U}, \hat{V}) \mid N \in\left\{1, \ldots, N_{\max }\right\} ; \delta \leq N\right. \\
&\left.\delta \in\left\{0, \ldots, \delta_{\max }\right\} ; \alpha \in\{-\beta, \beta\} ;(\hat{U}, \hat{V}) \neq \emptyset\right\}
\end{aligned}
$$

This set contains all viable control profiles that drive the system from the given condition at time 0 to the desired final condition $\equiv 0$ in time less or equal $N$.

2) Closed-loop Control Law: We will now proceed to define a closed-loop control algorithm for the system and state our main result.

\section{Algorithm 1:}

0 : Given the required initial parameters $T, u_{\max }, v_{\max }$, $\beta, O, p, q$ and the system current position; take an initial prediction horizon $t_{f}=N_{\max } T$ where:

$$
\begin{array}{r}
N_{\max }=\left\lceil\frac{1}{T} \max \left(\frac{\pi}{v_{\max }}, \frac{\pi r_{e}}{2\left(1-\sin \left(\frac{\beta T v_{\max }}{4}\right)\right) u_{\max }}\right)\right\rceil \\
+\left\lceil\frac{1}{T} \frac{d\left(\theta_{e}, \pi\right)}{\beta v_{\max }}\right\rceil
\end{array}
$$

Where $d(\cdot, \cdot)$ is an appropriate metric for $\mathbb{S}^{1}$, (in this case the smallest angle between $\theta_{e}$ and $\pi$ ); and $r_{e}=$ $\left\|\left(x_{e}, y_{e}\right)^{T}\right\|$. Also set:

$$
\delta_{\max }=\left\lceil\frac{\pi}{T \beta v_{\max }}\right\rceil
$$

1: Calculate the set of control profiles $\mathcal{C}$ as defined in (11). If $\mathcal{C}=\emptyset$ go back to Step 0 (for instance, if $\theta$ vanishes too quickly due to the effect of disturbances).

2: Define the "best" control profile $\left(\hat{U}_{o p t}, \hat{V}_{\text {opt }}\right)$ as the control profile $(\hat{U}, \hat{V}) \in \mathcal{C}$ that minimizes:

$$
J(\hat{U}, \hat{V})=q \hat{V}^{T} \hat{V}+p \hat{U}^{T} \hat{U}+\sum_{i=0}^{N-1} \zeta_{i}^{T} O \zeta_{i}
$$

With $O, p, q>0$.

3: The control law is given, at any time by applying the first element of $\hat{U}_{o p t}$ and $\hat{V}_{\text {opt }}$, reducing $\mathrm{N}$ by one (if $N>1$ ) and going back to Step 1 .

In order to treat the case of stabilization around a non-zero reference, a coordinate change such that $\mathbf{q}_{\text {ref }}^{\prime} \equiv 0$ has to be applied to the system.

Lemma 1: Choosing the initial prediction horizon from Step 0 of the algorithm; we will always find a nonempty $\mathcal{C}$ provided that $\beta T v_{\max }$ is neither zero nor a multiple of $2 \pi$.

Proof: We will invert the problem and characterize a region of the state space that can be reached in a time smaller or equal to the prediction horizon, and then show that it includes the reference. Within the limits of this proof, we will still consider $\theta_{\text {ref }} \equiv 0$ but take $\zeta_{0} \equiv 0$ instead.

Since we can reach, with our choice of $\delta_{\max }$ an angle at a distance of at most $\epsilon$ from $\pi$ (this error is in turn bounded by $\frac{\beta T v_{\max }}{2}$ since it is at most half a step of the initial turning phase). We shall consider that we start from $\pi$ at a time $(\delta+1) T$ and then observe the effects of the initial error in the range of the system.

From that point on, a control with no initial angle drift will follow; which implies a constant $v=\frac{-\Delta \theta}{T(N-\delta)}$.

At any time instant, we can define the radius of curvature of the path being followed by the cart as: $\rho_{k}=\frac{u_{k}}{v_{k}}$

For this value of $v$, let us analyze the paths that can be followed starting from the initial position ( $A$ in figure 1 ). We know the algorithm will steadily bring $\theta$ from $\pi$ to 0 with no overshoot (with $|u|$ constant); which in fact tells us that the followed path will have a length of $\pi \rho$, being $\rho$ the corresponding radius of curvature. Considering at most one inflection point in the sign of $u$, the resulting path can be thought of as following first an arc in a circle of radius $\rho$ tangent to the initial conditions of the vehicle subtending an angle $\phi\left(A E\right.$ or $A E^{\prime}$ in figure 1$)$; then changing to another circle, tangent to the first at the inflection point, and following it for an angle $\psi\left(E D\right.$ or $\left.E^{\prime} D\right)$. Here $\phi+\psi=\pi$, as clearly seen in the figure.

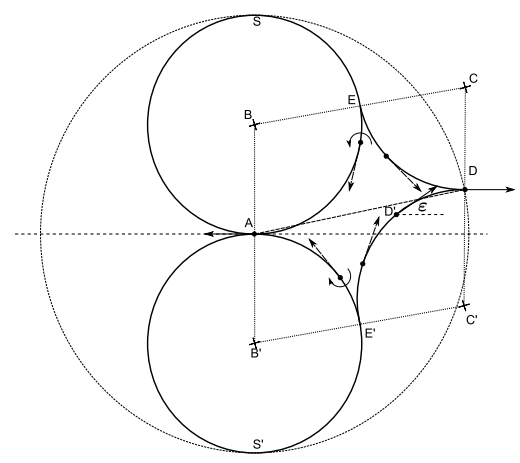

Fig. 1. Path respecting all the constraints and therefore belonging to $\mathcal{C}$.

We can compute for any curvature radius, with the given constant $v$, the time required to follow the path while respecting saturation constraints:

$$
t_{1}=T\left\lceil\frac{1}{T} \max \left(\frac{\pi}{v_{\max }}, \frac{\pi \rho}{u_{\max }}\right)\right\rceil
$$

Also, since the maximum time required to get to the necessary initial angle condition is when a turn of $\pi$ is performed, the time to reach the delta used in this proof is:

$$
t_{2}=T\left\lceil\frac{1}{T} \frac{d\left(\theta_{e}, \pi\right)}{\beta v_{\max }}\right\rceil
$$

In the figure it is clear that $B, E$ and $C$ are collinear (for $B$ and $C$ are the centers of two circles tangent at $E$ ) and that $A B$ is parallel to $C D$ (they are both vertical lines); therefore we can easily deduce that $|A D|=|B C|$ and, from analogous reasoning, equal also to $\left|B^{\prime} C^{\prime}\right|$. Since the distance between $B$ and $C$ is clearly twice the radius of the circles we can then say that the time required to get from point $A$ to any point $D$ that lies in a circle of radius $2 \rho$ by following this kind of path is the sum of both computed times.

Considering an initial distance of $\epsilon$ between the final obtained angle and the desired $\pi$ is analogous to considering 
the reference as forming an angle $\pm \epsilon$ with respect to the horizontal (as portrayed in figure 1 with the point $D^{\prime}$ ). By applying the triangle inequality, we know that $\left|A D^{\prime}\right| \geq$ $|A D|-\left|D D^{\prime}\right|$; and therefore we can bound the reachable radius in time $t_{1}+t_{2}$ by noting that $\left|D D^{\prime}\right|=2 \rho \sin \left(\frac{\epsilon}{2}\right)$ and $|A D|=2 \rho$.

Using the original distance from the reference and equating it to the reachable radius; the upper bound for the necessary time horizon that assures a suitable path to reach any point at a distance $\leq r_{e}$, and thus the reference, is found to be $N_{\max } T$.

Theorem 2: For the system defined in (1) and subject to saturation constraints $-u_{\max } \leq u \leq u_{\max },-v_{\max } \leq v \leq$ $v_{\text {max }}$; let $\mathbf{q}_{\text {ref }}$ be a desired final value in the configuration space of the system and $\mathbf{q}_{k}$ the state of the system at time $k T$. Then, Algorithm 1 defines a control law that stabilizes the system around $\mathbf{q}_{\text {ref }}$.

Proof: Taking Lemma 1, we will use $J\left(\hat{U}_{o p t}, \hat{V}_{o p t}\right)$ as a candidate Lyapunov function (where $\left(\hat{U}_{o p t}, \hat{V}_{o p t}\right)$ is the optimal path last chosen by the algorithm). We can appreciate that it is positive definite at all times and zero if and only if the final state can be reached without control action (meaning that we are already there and the error is zero). Furthermore, whenever the error is different from zero, the function is obviously strictly decreasing at all times; since from any time, and by following the same path as the previous instant, all the terms in $J\left(\hat{U}_{o p t}, \hat{V}_{\text {opt }}\right)$ at time $k+1$ were present at time $k$, plus a non-negative term (due to the quadratic nature of the weighting function). The only exception is when the algorithm chooses a different path, but it implies then that a smaller $J(\cdot)$ was found; therefore the function $J\left(\hat{U}_{o p t}, \hat{V}_{o p t}\right)$ is still strictly decreasing.

We can further say that $J\left(\hat{U}_{o p t}, \hat{V}_{o p t}\right)$ will reach zero in finite time following directly from Lemma 1 if the initial error was bounded. This proves the global asymptotic stability of the nominal system under the defined control law.

\section{DyNAMic MODELING FOR CONTROLLER VALIDATION}

In order to test the controller under simulation, a dynamic model of the system was developed by taking into account an approximation of Coulomb friction and point contact for the wheel-ground interaction, as well as first-order actuator dynamics. The model was also simplified by reducing the vehicle (originally four-wheeled) to a two-wheeled version, still based on skid-steering, see figure 2 . The center of gravity lies exactly between both wheels in the common axis.

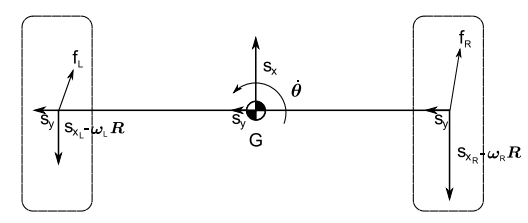

Fig. 2. Top view of the simplified system.

The Coulomb friction model was approximated as in [8] by a linear region with a high slope $\lambda$. As $\lambda$ goes to infinity the model behaves as Coulomb friction; otherwise, a small slippage is required to equilibrate external forces. The equation describing the friction coefficients (ratio between friction and normal forces at the contact point) is then:

$$
\mu(s)= \begin{cases}s \lambda & \text { if }|s \lambda| \leq \mu_{\max } \\ \mu_{\max } & \text { if }|s \lambda|>\mu_{\max }\end{cases}
$$

Where $s$ is the relative velocity of the contact surfaces, and $\mu_{\max }$ is the maximum value of the friction coefficient

The friction coefficients are computed with (14) where, for each wheel, $s$ is obtained as the resulting vector of velocities w.r.t. the ground in the $x_{r e l} G y_{\text {rel }}$ coordinate system. This velocity, for the right wheel is calculated as:

$$
\begin{aligned}
\left|s_{R}\right| & =\sqrt{\left(s_{x_{R}}-\omega_{R} R\right)^{2}+s_{y}^{2}} \\
s_{x_{R}} & =s_{x}+c \dot{\theta} \\
{\left[\begin{array}{l}
s_{x} \\
s_{y}
\end{array}\right] } & =\left[\begin{array}{cc}
\cos \theta & \sin \theta \\
-\sin \theta & \cos \theta
\end{array}\right]\left[\begin{array}{c}
\dot{x} \\
\dot{y}
\end{array}\right]
\end{aligned}
$$

Where $R$ is the radius of the wheels; $\omega_{R}$ is the angular velocity of the right wheel; $c$ is half the distance between the tires; and $\dot{x}, \dot{y}, \dot{\theta}$ are the current velocities of the system in global coordinates. The left wheel analysis is completely analogous. The corresponding friction forces acting on the left and right wheels $f_{L}$ and $f_{R}$ are computed by multiplying $\mu_{L}, \mu_{R}$ by the normal force.

The accelerations are, therefore:

$$
\left[\begin{array}{c}
\ddot{x} \\
\ddot{y} \\
\ddot{\theta}
\end{array}\right]=\left[\begin{array}{ccc}
\frac{1}{m} & 0 & 0 \\
0 & \frac{1}{m} & 0 \\
0 & 0 & \frac{1}{I}
\end{array}\right]\left[\begin{array}{c}
f_{R_{x}}+f_{L_{x}} \\
f_{R_{y}}+f_{L_{y}} \\
f_{R_{x}} c-f_{L_{x}} c
\end{array}\right]
$$

Where $m$ is the system mass and $I$ the moment of inertia around $G$. The accelerations are integrated to obtain the system velocities and integrated again to obtain the position.

Control variables $u$ and $v$ from section II are translated to desired angular velocities for the wheels as follows:

$$
\omega_{R_{d e s}}=\frac{u+c v}{R} \quad \omega_{L_{d e s}}=\frac{u-c v}{R}
$$

First order actuator dynamics are considered for the tracking of these desired angular velocities, product of a low-level controller at each wheel which will not be addressed here.

\section{Simulation Results}

The control algorithm was implemented in Matlab and tested on the proposed dynamic model. The controller parameters for the different cases were obtained by tuning the parameter in order to obtain good performances.

The implementation of the algorithm incorporates a tolerance level below which, no control action will be taken. This is, when:

$$
\mathbf{q}_{e}^{T} \mathbf{W} \mathbf{q}_{e}<\epsilon
$$

Where $\mathbf{W}$ is a weighting matrix. In this case, for validation purposes, we have selected $W=\left[\begin{array}{ccc}100 & 0 & 0 \\ 0 & 100 & 0 \\ 0 & 0 & 10\end{array}\right]$; and $\epsilon=0.001$. This means that we accept a maximum error of approximately $3[\mathrm{~mm}]$ in position or $0.6[\mathrm{deg}]$ in orientation. 


\begin{tabular}{|c|c|}
\hline Kinematic & $\begin{array}{c}a=b=0.25[\mathrm{~m}] \\
c=0.20[\mathrm{~m}], R=0.108[\mathrm{~m}]\end{array}$ \\
\hline Dynamic & $m=14[\mathrm{~kg}], I=0.07\left[\mathrm{~kg} \cdot \mathrm{m}^{2}\right]$ \\
\hline Friction & $\begin{array}{l}\lambda=1000, \mu_{\max }=0.6 \\
\text { (rubber on wet asphalt) }\end{array}$ \\
\hline Actuators & $\begin{array}{c}u_{\max }=0.56[\mathrm{~m} / \mathrm{s}], \\
v_{\max }=0.56[\mathrm{rad} / \mathrm{s}], \\
\tau=0.0625[\mathrm{~s}]\end{array}$ \\
\hline Regulation & $\begin{array}{c}p=q=1, O=\left[\begin{array}{cc}0.5 & 0 \\
0 & 0.5\end{array}\right], \\
\beta=0.5, T=1.5[s]\end{array}$ \\
\hline
\end{tabular}

TABLE I

PARAMETERS USED FOR THE SIMULATIONS

\section{A. Stabilization}

Case 1: For the first proposed scenario with the dynamic model, the initial position of the robot is $\mathbf{q}_{0}=(0,3,0)^{T}$ and it should, of course, reach the position $\mathbf{q}_{\text {ref }}=(0,0,0)^{T}$. This is a difficult case since the reference is completely parallel to the initial condition. The trajectories followed by both the kinematic and dynamic models of the vehicle can be seen in Figure 3(a). The difference between these trajectories is quite small. The steady state errors are found to be $1.7[\mathrm{~mm}]$ in $x, 0[\mathrm{~mm}]$ in $y$ and $3.7 \times 10^{-4}[\mathrm{rad}]$ in $\theta$; which fall within accepted levels. The final state is reached in approximately 31.5 seconds, as seen in Figure 3(b) and (c). Notice in Figure 3(d) that the control values remain within the preestablished limits at all times. The cost function, as appreciated in Figure 3(e), is always decreasing, as expected. Case 2: Also, in Figure 4, the capacity of the regulator to stabilize the system around a non-zero reference was tested. The initial posture was $\mathbf{q}_{0}=(0,1,0)^{T}$ and the reference was chosen as $\mathbf{q}_{f}=\left(-0.5,-0.5,-\frac{2 \pi}{3}\right)^{T}$. In Figure 4(a), a topview of the trajectory followed by the system is presented. In this case, the final position was reached in $21.2[s]$, as seen in 4(b) and (c), while the final errors in position were $1.2[\mathrm{~mm}]$ in $x, 2.1[\mathrm{~mm}]$ in $y$ and $9 \times 10^{-4}[\mathrm{rad}]$ in theta; again within tolerated levels. In Figure 4(d), control values are inside the permitted range and the cost function in 4(e) is decreasing.

\section{Conclusions}

In this paper, a Model Predictive Control scheme was proposed for a Skid-Steering Mobile Robot permitting the asymptotic stabilization of the system around an arbitrary reference position. Furthermore, asymptotic stability of the nominal system under this scheme was analytically proven, as well as the existence of solutions in finite time for bounded position errors and non-zero saturation levels by using an approach based on the specific choice of the angular velocity profile. The computational cost was reduced by separating the system into two subsystems before solving the (convex) optimization problem.

As seen in simulation results, the overall performance of the controller is comparable to that obtained by the use of a kinematic oscillator (see for instance [10]) but with the advantages of incorporating actuator saturation constraints and energetic considerations into the computation of the system trajectory. Robustness of the controller w.r.t. unmodeled dynamics was tested under simulation with both the ideal kinematic model and a more complete dynamic one.

\section{REFERENCES}

[1] M. Alamir and N. Marchand, Constrained minimum-time-oriented feedback control for the stabilization of nonholonomic systems in chained form, Journal of Optimization Theory and Applications 118 (2003), 229-244.

[2] A. Astolfi, Exponential stabilization of a wheeled mobile robot via discontinuous control, Journal of Dynamic Systems, Measurements and Control 121 (1999), 121-126.

[3] Salim Belkhous, Adel Azzouz, Maarouf Saad, Chahe Nerguizian, and Vahe Nerguizian, A novel approach for mobile robot navigation with dynamic obstacles avoidance, Journal of Intelligent and Robotic Systems (2005).

[4] R. W. Brockett, Asymptotic stability and feedback stabilization, Differential Geometric Control Theory, 1983, pp. 181-191.

[5] Ahmed Chemori and Nicolas Marchand, Global discrete time stabilization of the PVTOL aircraft based on a fast predictive controller, IFAC World Congress, 2008.

[6] S. Jakubek, M. Seyr, and G. Novak, Autonomous mobile robot propioceptive navigation and predictive trajectory tracking, International Journal of Control 81 (2008), 989-1001.

[7] Yutaka Kanayama, Yoshihiko Kimura, Funio Miyazaki, and Tetsuo Noguchi, A stable tracking control method for an autonomous mobile robot, Proceedings of the IEEE International Conference on Robotics and Automation, 1990.

[8] Ryo Kikuuwe, Naoyuki Takesue, Akihito Sano, Hiromi Mochiyama, and Hideo Fujimoto, Fixed-step friction simulation: From classical coulomb model to modern continuous models, International conference on intelligent robots and systems, 2005.

[9] Ilya Kolmanovsky and N. Harris McClamroch, Developments in nonholonomic control problems, IEEE Control Systems (1995), 20-36.

[10] Krzysztof Kozlowski and Dariusz Pazderski, Modeling and control of a 4-wheel skid-steering mobile robot, International Journal of Applied Mathematics and Computer Science 14 (2004), 477-496.

[11] David A. Lizrraga, Obstructions to the existence of universal stabilizers for smooth control systems, Mathematics of Control, Signals, and Systems 16 (2003), 255-277.

[12] Alessandro De Luca and Giuseppe Oriolo, Modeling and control of nonholonomic mechanical systems, Kinematics and dynamics of multibody systems, 1995, pp. 277-342.

[13] Elie Maalouf, Maarouf Saad, and Hamadou Saliah, A higher level path tracking controller for a four-wheel differentially steered mobile robot, Robotics and Autonomous Systems 54 (2006), 23-33.

[14] Nicolas Marchand and Mazen Alamir, Discontinuous exponential stabilization of chained form systems, Automatica 39 (2003), 343348.

[15] Dariusz Pazderski, Krzysztof Kozlowski, and W. E. Dixon, Tracking and regulation control of a skid steering vehicle, ANS tenth international topical meeting on robotics and remote systems, 2004.

[16] G. Poulin, A. Chemori, and N. Marchand, Minimum energy oriented global stabilizing control of the PVTOL aircraft, International Journal of Control 80 (2007), 430-442.

[17] Arthur Richards and Jonathan P. How, Model predictive control of vehicle maneuvers with guaranteed completion time and robust feasibility, Proceedings of the American Control Conference, 2003, pp. 4034-4040.

[18] Eduardo D. Sontag, Mathematical control theory, deterministic finite dimensional systems, 2nd ed., Springer-Verlag, 1998.

[19] Stability and stabilization: Discontinuities and the effect of disturbances, Proceedings of NATO Advanced Study Institute: Nonlinear Analysis, Differential Equations, and Control, 1998, pp. 551597.

[20] Amel Zerigui, Xiang Wu, and Zong-Quan Deng, A survey of rover control systems, IJCSES International Journal of Computer Sciences and Engineering Systems Vol.1, No.2 (2007), 105-109. 


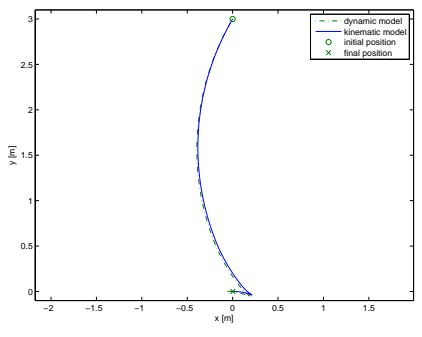

(a)

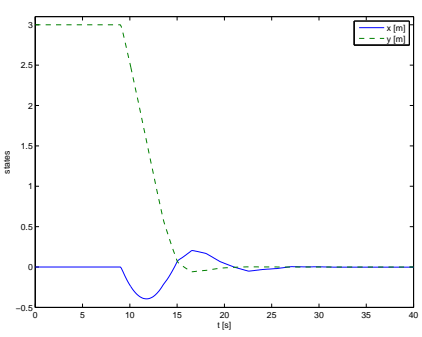

(b)

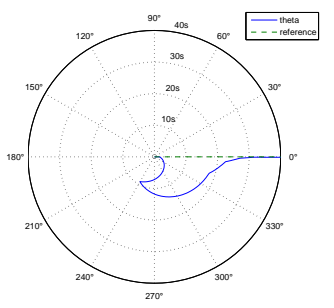

(c)

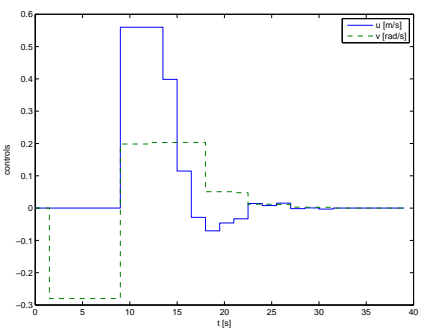

(d)

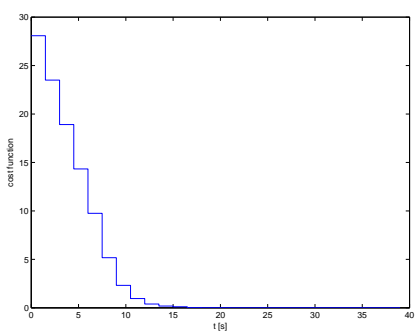

(e)

Fig. 3. Stabilization around zero: (a) Top view of the trajectory followed by the dynamic and kinematic models; (b) Evolution of the $x$ and $y$ positions over time; (c) Evolution of the orientation angle; (d) Applied control signals; (e) Evolution of the cost function $J\left(\hat{U}_{o p t}, \hat{V}_{o p t}\right)$.

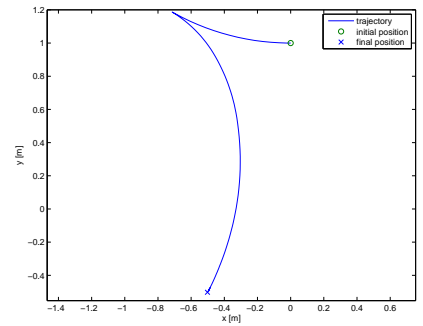

(a)

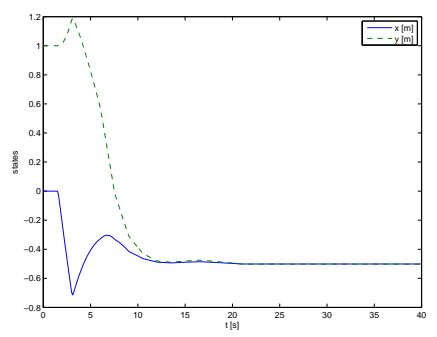

(b)

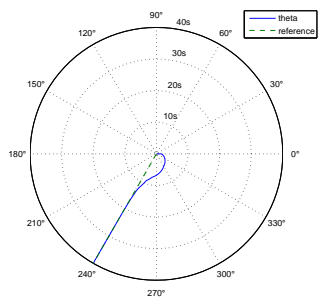

(c)

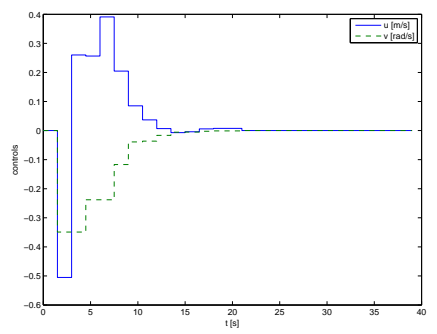

(d)

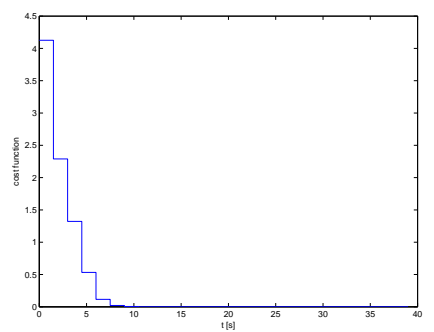

(e)

Fig. 4. Stabilization around a non-zero reference: (a) Top view of the trajectory followed by the dynamic model; (b) Evolution of the $x$ and $y$ positions over time; (c) Evolution of the orientation angle; (d) Applied control signals; (e) Evolution of the cost function $J\left(\hat{U}_{o p t}, \hat{V}_{o p t}\right)$. 